\title{
Multiplateau structure in photoemission spectra of strong-field ionization of dense media
}

\author{
M. Wilke, R. Al-Obaidi, I. Yu. Kiyan, ${ }^{*}$ and E. F. Aziz ${ }^{\dagger}$ \\ Joint Ultrafast Dynamics Lab in Solutions and at Interfaces (JULiq), Helmholtz-Zentrum Berlin, Albert-Einstein-Str. 15, \\ D-12489 Berlin, Germany \\ and Freie Universität Berlin, FB Physik, Arnimallee 14, D-14195 Berlin, Germany
}

(Received 10 June 2016; published 29 September 2016)

\begin{abstract}
Strong-field ionization of dense molecular gases in a short infrared laser pulse is studied by means of photoelectron spectroscopy combined with a liquid microjet technique. By increasing the gas density, we observe how the laser-assisted electron scattering on neighboring particles becomes a dominant mechanism of hot electron emission. The angle-resolved energy distributions of rescattered electrons are obtained by analyzing the density dependency of emission spectra. A semiclassical consideration of electron trajectories is shown to provide a good description of experimental spectra. The model predicts the existence of four energy plateaus. Two cutoffs at higher energies are evident in the spectra.
\end{abstract}

DOI: 10.1103/PhysRevA.94.033423

Following the development of powerful laser systems of short pulse duration, interaction of matter with strong laser fields has received wide interest during the last decades. Ionization represents one of the fundamental phenomena occurring in the laser field. This phenomenon was studied in detail on the atomic (molecular) level, corresponding to ionization of diluted gases [1,2]. Emission of hot electrons is a characteristic feature of strong-field ionization. This effect, known as above-threshold ionization (ATI), occurs due to absorption of a number of photons in excess of the minimum number needed to overcome the ionization threshold. Driven by the external laser field, the released electron can revisit the parent core and absorb even more energy from the field during the rescattering event, giving rise to high-order ATI (HATI). Both ATI and HATI processes have received a detailed consideration. The theory based on the strong-field approximation (SFA) [3-5], where the electron interaction with the core in the final state is neglected, was demonstrated to describe well ATI and HATI of atomic systems $[6,7]$. Recently, the SFA theory was extended to describe these processes in molecules $[8,9]$, which require a more complex consideration. The semiclassical analysis of electron trajectories yields the energy cutoffs of $2 U_{p}$ and $10 U_{p}$ for ATI and HATI, respectively [10]. Here $U_{p}=F^{2} / 4 \omega^{2}$ is the electron ponderomotive energy in a linearly polarized laser field of frequency $\omega$ and strength $F$ (atomic units are used throughout).

In a dense medium, the ATI process can be followed by scattering of the released electrons on neighboring molecules before the laser pulse is passed through the medium. Similar to HATI, the photoelectron can gain additional energy from the laser field via the process of laser-assisted electron scattering (LAES) [11-15]. However, this ionization channel has incoherent character because the path of released electron to a neighboring particle is random. Below we refer to the ATI process followed by LAES as incoherent HATI (iHATI).

While a comprehensive description of iHATI is presented in literature [16], experimentally this effect is addressed to

\footnotetext{
*igor.kiyan@helmholtz-berlin.de

†emad.aziz@helmholtz-berlin.de
}

a lesser extent. In our first observation of iHATI [17], we found that it becomes the dominant mechanism of hot electron emission at media densities exceeding $10^{15} \mathrm{~cm}^{-3}$. This is an important issue for the efficiency of plasma heating $[18,19]$. The iHATI can also play a role as a secondary effect in generation of soft $\mathrm{x}$ rays via laser frequency upconversion induced in a partially ionized dense gas medium [20]. As shown in Ref. [21], laser-assisted radiative recombination can lead to emission of $\mathrm{x}$ rays with photon energies well above the cutoff of high-order harmonic generation, where the photoelectron is scattered on the parent ion. Very recently, efficient generation of hot electrons from clusters exposed to an infrared laser pulse of ultrashort duration was reported [22]. The ionization yield in the plateau energy range of HATI was found to be enhancement by orders of magnitude as compared to ionization of isolated atoms. Apparently, the iHATI process needs to be considered to interpret this effect.

In view of the fundamental importance of iHATI, in the present work we provide a direct experimental study of this effect. Angle-resolved energy distributions of electrons generated in the iHATI process are obtained by analyzing the dependency of photoemission spectra on the density of gas (vapor) used as the interaction medium. Our study demonstrates the existence of several energy cutoffs in emission spectra generated by the iHATI process. Calculations based on semiclassical analysis of electron trajectories will be shown to reproduce well the experimental iHATI spectra.

A detailed description of the experimental setup can be found in Ref. [17]. Briefly, linearly polarized infrared laser pulses of $1.3 \mu \mathrm{m}$ wavelength were generated in an optical parametric amplifier (OPA) pumped with a Ti:sapphire laser system at a repetition rate of $5 \mathrm{kHz}$. The pulses were focused by a spherical lens in front of the time-of-flight (TOF) electron spectrometer, with the TOF axis positioned perpendicular to the laser beam propagation axis. The focal spot size of $20 \mu \mathrm{m}$ (FWHM) and the pulse duration of $38 \mathrm{fs}$ (FWHM), corresponding to eight optical cycles, were measured with the use of the beam diagnostic tools. The laser beam was attenuated in order to prevent saturation of the electron detector by the large amount of electrons generated in dense medium. The peak intensity in the laser focus was adjusted to $5 \times 10^{13} \mathrm{~W} / \mathrm{cm}^{2}$, yielding a ponderomotive energy of 
approximately $9 \mathrm{eV}$. The laser polarization axis was controlled by a $\lambda / 2$ wave plate, enabling one to record angle-resolved photoelectron spectra. The emission angle $\theta$ is defined as the angle between the laser polarization and the spectrometer axis.

Dense vapors of water and acetone were used as the target medium. To facilitate the high-vacuum conditions required for electron detection, a liquid microjet technique [23] was applied to create a liquid jet of $20 \mu \mathrm{m}$ size in the vicinity of the laser focus. Due to the cylindrical symmetry of the liquid jet, the vapor pressure in the interaction region is defined by $[17,23]$

$$
P=\frac{R_{0}}{R} P_{0} \quad\left(R \geqslant R_{0}\right),
$$

where $P_{0}$ is the equilibrium vapor pressure at the jet surface, $R_{0}=10 \mu \mathrm{m}$ is the jet radius, and $R$ is the distance between the jet center and the laser focus. By changing the jet position with respect to the laser focus, the target vapor pressure was varied by several orders of magnitude, between $P_{0}$ and the residual gas pressure in the experimental chamber $\left(\sim 10^{-4}\right.$ mbar $)$. The equilibrium vapor pressure of acetone and water at $0^{\circ} \mathrm{C}$ temperature are 100 and 6 mbar, respectively. Thus, with the use of acetone higher gas densities could be reached in the laser focus. A small $(20 \mathrm{mM})$ concentration of $\mathrm{NaI}$ was added to both water and acetone to prevent a streaming potential of the liquid flow [24].

The design and performance characteristics of the TOF spectrometer are presented in detail in Ref. [25]. The spectrometer was operated in the field-free configuration, yielding the electron acceptance angle of $1^{\circ}$. This value defines the angular resolution in the present experiment. The spectrometer entrance was equipped with a skimmer of $200 \mu \mathrm{m}$ orifice, used to facilitate differential pumping of the TOF region. The orifice size defines the electron acceptance length of the interaction region. Since it is much smaller than the Rayleigh length of $2 \mathrm{~mm}$ of the laser focus, the variation of laser intensity along the beam axis will be disregarded in the data analysis. A low energy filter, consisting of a grid biased at negative potential, was applied in front of the detector to prevent detection of the large amount of slow electrons generated in the ATI process. A typical bias voltage of $-50 \mathrm{~V}$ enabled one to keep the electron count rate below the saturation limit of the detection electronics. The spectral range above the energy of $50 \mathrm{eV}$ is of primary interest in this study. In this range, the HATI and iHATI processes contribute to the ionization yield and, depending on the gas density, compete with each other. It is also important to note that the high-energy part of the spectrum is not affected by the space charge effect because the fast electrons leave the interaction region before a significant charge is cumulated.

A series of angle-resolved photoelectron spectra were recorded for different densities of acetone and water. Two representative emission spectra, obtained with low and high acetone vapor pressure of $10^{-1}$ and 20 mbar, respectively, are shown in Figs. 1(a) and 1(b). Ionization in the diluted-gas limit [see Fig. 1(a)] gives rise to a typical HATI spectrum, exhibiting a narrow angular distribution of photoelectrons along the laser polarization axis with a cutoff energy of approximately $10 U_{p}$. This distribution undergoes tremendous changes with the increase of the gas density [see Fig. 1(b)]. First, the HATI cutoff energy becomes abandoned and a photoelectron signal at kinetic energies exceeding $18 U_{p}$ arises in the spectrum.

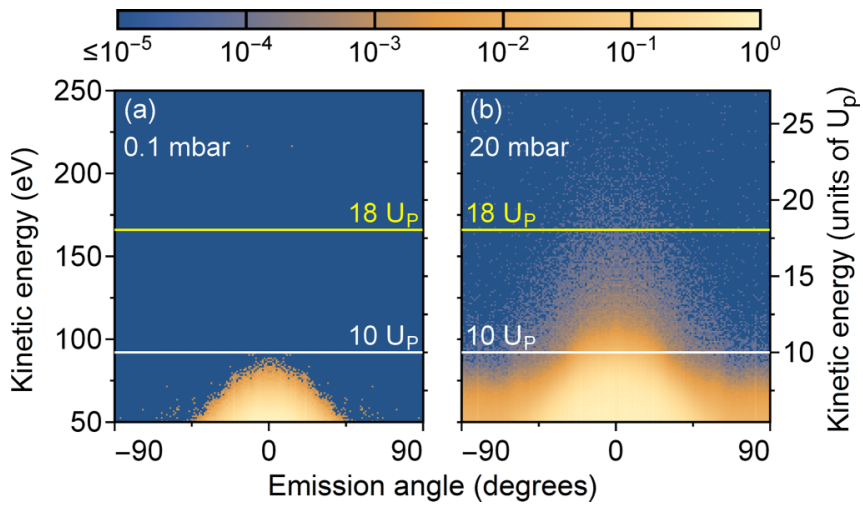

FIG. 1. Angle-resolved emission spectra obtained for acetone at (a) low (10 $\left.{ }^{-1} \mathrm{mbar}\right)$ and (b) high (20 mbar) vapor pressure. The HATI and iHATI processes contribute to the ionization yield in the shown energy range above $50 \mathrm{eV}$. Horizontal lines indicate kinetic energies of $10 U_{p}$ (HATI cutoff) and $18 U_{p}$ (iHATI highest-energy cutoff; see text).

Secondly, the angular distribution becomes broadened. The broadening is more remarkable at lower kinetic energies, where ionization yield perpendicular to the laser polarization axis is prominent up to $\sim 10 U_{p}$ energies. As shown below, these spectral features are due to the iHATI process which dominates ionization of the dense medium in the considered kinetic energy range.

Contrary to the data analysis used in our previous work [17], where the total ionization yield was investigated as a function of gas density, in the present study we follow the density dependency separately for a set of kinetic energies and emission angles, taking into account the energy resolution of $\sim 1 \mathrm{eV}$ [25] and the angular resolution of $1^{\circ}$. This routine allows one to decompose emission spectra into the individual contributions of the HATI and iHATI processes, according to their $P$ and $P^{2}$ dependencies, respectively. The accuracy of such a decomposition relies on a significantly higher resolution of the vapor pressure as compared to our previous experiment [17].

A typical dependency of the differential yield on the distance between laser focus and liquid microjet is shown in Fig. 2 for the case of ionization of water. For the sake of demonstration, the emission signal was integrated over a larger section in the spectra, encompassing the energy range between 85 and $150 \mathrm{eV}$ and emission angles between $10^{\circ}$ and $20^{\circ}$. The dependency reveals ionization of three different phases: diluted gas, dense gas, and liquid. The latter gives rise to a stepwise increase of the signal by a few orders of magnitude, when the jet is moved into the laser focus. Ionization of the liquid phase lies, however, beyond the scope of the present work. As one should expect [17], ionization of the dilute and dense gas phase exhibits the $P$ and $P^{2}$ dependencies, respectively. Similar results were obtained for the differential ionization yield of acetone. In general, the $P^{2}$ dependency is stronger pronounced in the experiment on acetone because of the possibility to reach higher vapor densities.

The $P^{2}$ dependency is used in this work to fingerprint the angle-resolved iHATI spectrum. For each kinetic energy $E$ and emission angle $\theta$, the signal dependency on the pressure $P$ was 


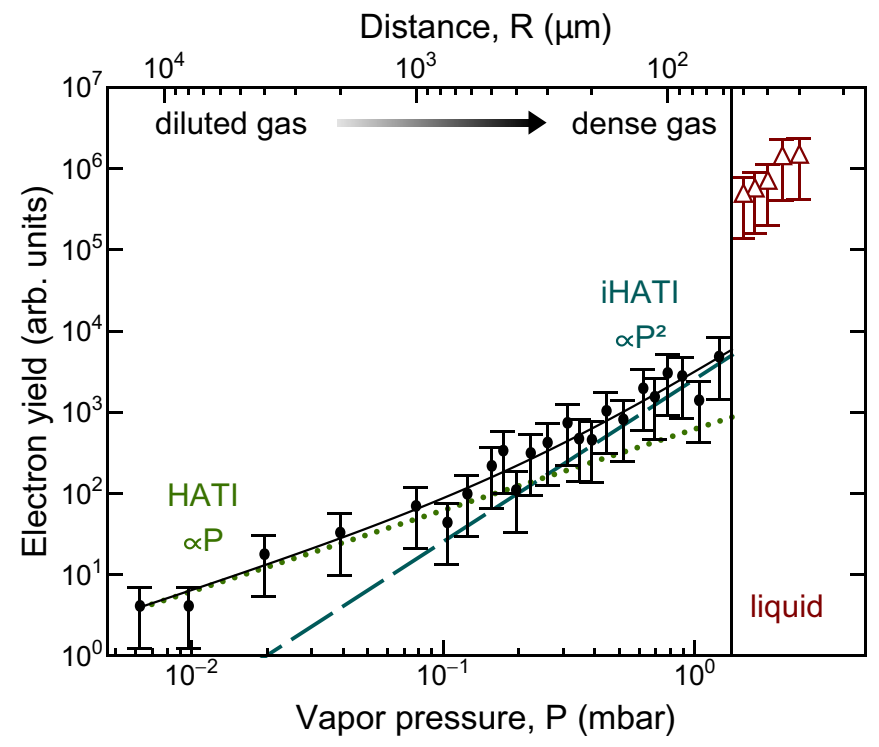

FIG. 2. Ionization yield of water versus the distance between laser focus and microjet (top scale). The yield is integrated over the range of electron kinetic energies from 85 to $150 \mathrm{eV}$ and emission angles $10^{\circ} \leqslant \theta \leqslant 20^{\circ}$. The liquid-phase signal is marked by red triangles on the right-hand side. Ionization of gas phase exhibits the linear (dotted green line) and quadratic (dashed cyan line) dependency in the limit of dilute and dense vapor, respectively. A fit to a combination of these dependencies is shown by the solid black line. The gas pressure (bottom scale) is calculated according to Eq. (1).

fitted to the function $P S_{1}(E, \theta)+P^{2} S_{2}(E, \theta)$. Here $S_{1}$ and $S_{2}$ are the HATI and iHATI spectral amplitudes, respectively, which were treated as fit parameters. The result of the emission decomposition to the HATI and iHATI spectra, obtained for acetone, is shown in Fig. 3. The decomposed HATI spectrum [see Fig. 3(a)] appears very similar to the spectrum in Fig. 1(a) obtained for diluted gas. Figure 3(b) shows an experimental emission spectrum of the iHATI process.

Below we analyze the iHATI spectrum in terms of semiclassical electron trajectories by employing the theory presented in Refs. [16,26]. The energy conservation condition for

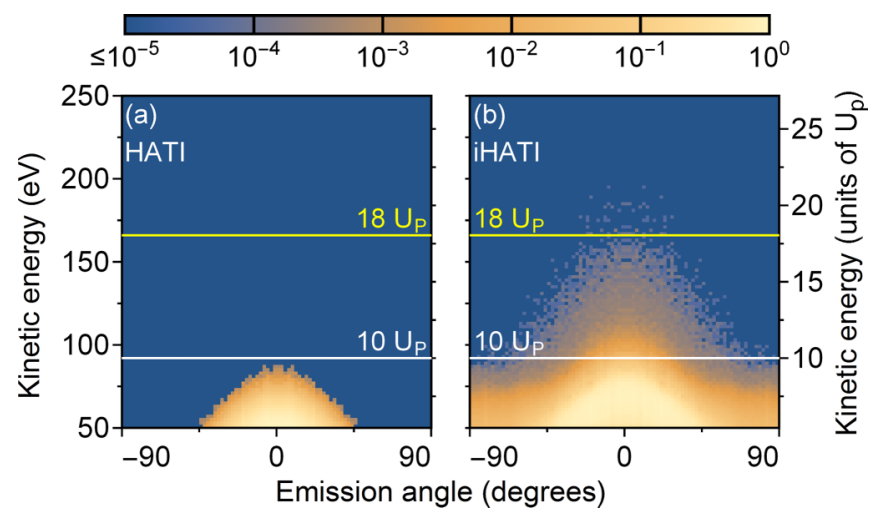

FIG. 3. Decomposition of the photoemission signal to the (a) HATI and (b) iHATI spectra according to the linear and the square dependency, respectively, of the differential yield on the gas pressure. The results are shown for acetone. laser-assisted scattering of an ionized electron on a neighboring particle has the form:

$$
\left[\boldsymbol{k}_{i}+\boldsymbol{A}(t)\right]^{2}=\left[\boldsymbol{k}_{f}+\boldsymbol{A}(t)\right]^{2},
$$

where $\boldsymbol{k}_{i}$ and $\boldsymbol{k}_{f}$ are the incident and final electron momenta, respectively, and $\boldsymbol{A}(t)=A_{0} \cos (\omega t) \hat{\boldsymbol{\epsilon}_{L}}$ is the vector potential of the linear polarized monochromatic field with $\hat{\epsilon_{L}}$ being the unit vector. The solution of Eq. (2) yields the final energy $E_{f}$ of the scattered electron [16]:

$$
E_{f}=4 U_{p}\left(a^{2} b^{2}+\beta a+\frac{\beta^{2}}{2} \pm a b \sqrt{a^{2} b^{2}+\beta^{2}+2 \beta a}\right),
$$

where $a=\cos \theta_{i} \cos \omega t, b=\cos \theta_{f} / \cos \theta_{i}, \beta=k_{i} / A_{0}$, and $\theta_{i, f}$ are the polar coordinates of $\boldsymbol{k}_{i, f}$. As shown below, Eq. (3) gives rise to four characteristic roots in the angle-resolved spectrum of iHATI electrons (labeled I-IV), defined by the sign of $a$ and $b$. This implies that the orientation of the incident momentum with respect to the external field vector and the mutual orientation of the incident and scattered electron momenta are crucial parameters for the LAES event.

Considering different electron trajectories, characterized by $\boldsymbol{k}_{i}, \boldsymbol{k}_{f}$, and $\phi=\omega t$ and satisfying the condition (3), and taking into account the intensity distribution in the laser focus, the iHATI electron spectrum is represented by

$$
S_{2}\left(E_{f}, \theta_{f}\right) \propto \int_{0}^{I_{0}} f(I) \int_{\boldsymbol{k}_{i}, \phi} w_{I}\left(\boldsymbol{k}_{i}, \boldsymbol{k}_{f}, \phi\right) d \boldsymbol{k}_{i} d \phi d I,
$$

where $f(I) \propto \sqrt{\ln \left(I_{0} / I\right)} I_{0} / I$ is the intensity distribution of a Gaussian pulse [27], $I_{0}$ denotes the peak intensity, and $w_{I}$ is the intensity-dependent weight of trajectories defined by the momentum distribution of ATI electrons and the probability of the LAES transition.

Making a simple approximation, we disregard quantum effects and consider $w_{I}$ to be independent of the trajectory parameters. Integration over $k_{i}$ is carried out in a range limited to $\sqrt{I} / \omega$, corresponding to the semiclassical energy cutoff of $2 U_{p}$ of ATI electrons. Although this approximation appears rather crude, it provides a good qualitative description of the observed iHATI spectra, as demonstrated below. It was pointed out before $[16,26]$ that quantum consideration of the LAES and ATI processes yields a rather smooth iHATI spectrum, where effects of quantum interference do not arise. The spectrum is also smoothed due to the intensity distribution. Therefore, merely scaling of contributions from different roots of Eq. (3), giving rise to different cutoff energies, is sufficient to reproduce the experimental spectrum. These contributions are shown in Fig. 4 for the case of negative sign on the right-hand side of Eq. (3). In the case of positive sign, identical spectra are obtained with the opposite sign of $b$. One can see that each root (I-IV) exhibits a characteristic plateau structure. The corresponding cutoff energies of $18,12,6$, and $2 U_{p}$ can be easily derived from Eq. (3). The highest cutoff energy of $18 U_{p}$ can also be obtained from Eq. (14) of Ref. [16] by considering scattering of ATI electrons with $2 U_{p}$ energy. Except for the root IV, Fig. 4 demonstrates a substantial energy gain of ATI electrons due to the LAES process.

The predicted multiplateau structure is well pronounced in the measured iHATI spectra of acetone and water, as 


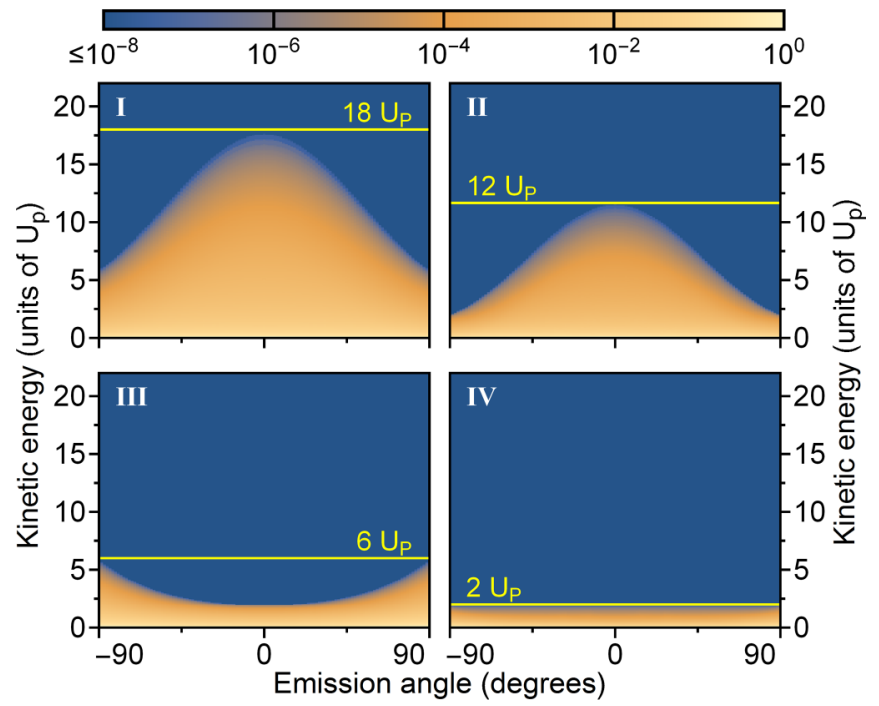

FIG. 4. Emission spectra of the iHATI process simulated separately for the four branches of electron trajectories: (I) $[a>0, b>0]$; (II) $[a<0, b<0]$; (III) $[a>0, b<0]$; (IV) $[a<0, b>0]$, where $a$ and $b$ are introduced in Eq. (3). Each branch gives rise to a characteristic emission plateau with a cutoff energy of 18, 12, 6, and $2 U_{p}$, respectively.

demonstrated in Fig. 5 which shows energy distributions of iHATI electrons for two chosen ranges of the emission angle $\theta_{f}$. The energy scale covers the cutoff appearance for roots I, II, and III. One can see that the simulated contribution of roots I and II reproduces well the acetone spectrum, including the two cutoff energies. As mentioned above, the iHATI signal is much weaker for water and, therefore, the root-I contribution cannot be distinguished from the background in the water spectrum. In Fig. 5, the water spectrum is scaled to reproduce the maximum signal of acetone and the higher-energy part, containing only background, is not shown. It is remarkable that the emission spectra of water and acetone exhibit rather similar shape, revealing a general character of the iHATI process.

Summarizing, this work provides an experimental study of the strong-field ionization process in dense media, which involves direct electron emission followed by laser-assisted scattering on neighboring particles. Similar to the well-known HATI process in a single atom or molecule, this incoherent ionization channel gives rise to emission of hot electrons and exhibits several characteristic plateaus with cutoff energies

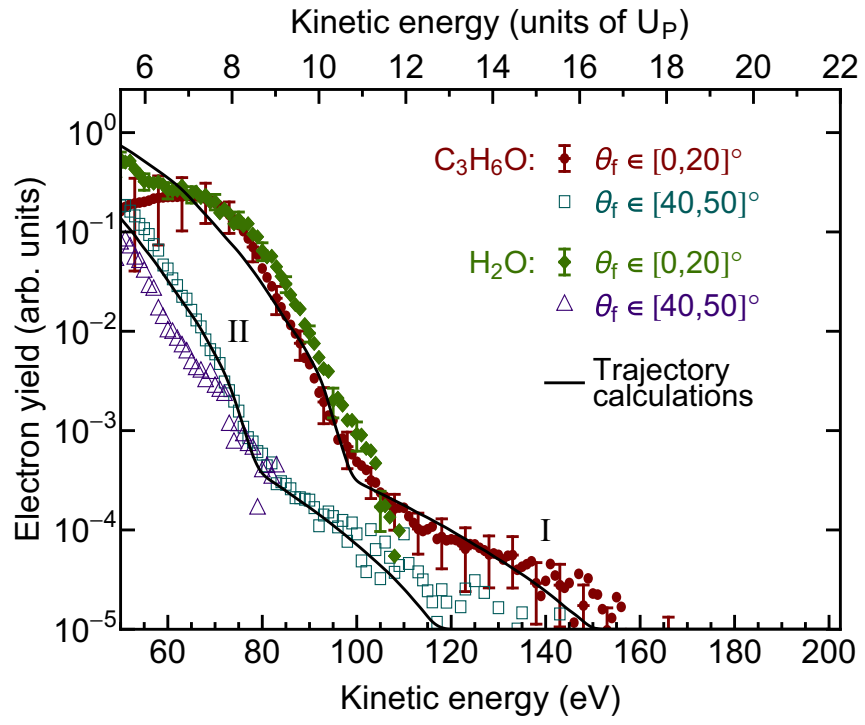

FIG. 5. The iHATI yield of water $\left(\mathrm{H}_{2} \mathrm{O}\right)$ and acetone $\left(\mathrm{C}_{3} \mathrm{H}_{6} \mathrm{O}\right)$ integrated over two chosen ranges of the emission angle $\theta_{f}$. Solid lines show the simulated yield of roots I and II (see Fig. 4) fitted to acetone spectra. The presence of two plateaus, characterized by cutoff energies of 12 and $18 U_{p}$ at $\theta=0^{\circ}$, is well pronounced in experiment and is reproduced by simulations. The water yield is scaled to the maximum of the acetone signal.

of $18,12,6$, and $2 U_{p}$. The two higher-energy cutoffs are clearly observed in the present experiment. At high densities of the interaction medium, generation of hot electrons is dominated by the iHATI process. A simple consideration of semiclassical electron trajectories, without taking quantum effects into account, is sufficient to reproduce the main features of angle-resolved iHATI spectra. The similarity of emission spectra of water and acetone points to a general character of this process. We believe that our findings not only provide a deeper understanding of elementary strong-field processes, but are also highly relevant to other research areas such as cluster physics, plasma physics, laser-driven generation of $x$ rays, and laser processing.

This work is funded by the European Research Council, Grant No. 279344, and by the Helmholtz-Gemeinschaft via the VH-NG-635 Grant.
[1] L. Dimauro and P. Agostini, Adv. At. Mol. Opt. Phys. 35, 79 (1995).

[2] W. Becker, F. Grasbon, R. Kopold, D. B. Milošević, G. Paulus, and H. Walther, Adv. At. Mol. Opt. Phys. 48, 35 (2002).

[3] L. V. Keldysh, ZhETF 47, 1945 (1965) [J. Exp. Theor. Phys. 20, 1307 (1965)].

[4] G. F. Gribakin and M. Y. Kuchiev, Phys. Rev. A 55, 3760 (1997).

[5] D. B. Milošević, G. G. Paulus, D. Bauer, and W. Becker, J. Phys. B 39, R203 (2006).

[6] B. Bergues and I. Y. Kiyan, Phys. Rev. Lett. 100, 143004 (2008).
[7] A. Gazibegović-Busuladžić, D. B. Milošević, W. Becker, B. Bergues, H. Hultgren, and I. Y. Kiyan, Phys. Rev. Lett. 104, 103004 (2010).

[8] M. Okunishi, R. Itaya, K. Shimada, G. Prümper, K. Ueda, M. Busuladžić, A. Gazibegović-Busuladžić, D. B. Milošević, and W. Becker, Phys. Rev. Lett. 103, 043001 (2009).

[9] M. Li, X. Sun, X. Xie, Y. Shao, Y. Deng, C. Wu, Q. Gong, and Y. Liu, Sci. Rep. 5, 8519 (2015).

[10] G. G. Paulus, W. Becker, W. Nicklich, and H. Walther, J. Phys. B 27, L703 (1994).

[11] N. M. Kroll and K. M. Watson, Phys. Rev. A 8, 804 (1973). 
[12] F. Bunkin and M. Fedorov, ZhETF 49, 1215 (1966) [J. Exp. Theor. Phys. 22, 844 (1966)].

[13] R. Kanya, Y. Morimoto, and K. Yamanouchi, Phys. Rev. Lett. 105, 123202 (2010).

[14] A. Čerkić and D. B. Milošević, Phys. Rev. A 87, 033417 (2013).

[15] A. N. Zheltukhin, A. V. Flegel, M. V. Frolov, N. L. Manakov, and A. F. Starace, J. Phys. B 48, 075202 (2015).

[16] A. Čerkić and D. B. Milošević, J. Phys. B 39, 4419 (2006).

[17] M. Wilke, R. Al-Obaidi, A. Moguilevski, A. Kothe, N. Engel, J. Metje, I. Y. Kiyan, and E. F. Aziz, New J. Phys. 16, 083032 (2014).

[18] S. C. Rae and K. Burnett, Phys. Rev. A 46, 1084 (1992).

[19] T. Ditmire, J. Zweiback, V. P. Yanovsky, T. E. Cowan, G. Hays, and K. B. Wharton, Nature (London) 398, 489 (1999).

[20] T. Popmintchev, M.-C. Chen, D. Popmintchev, P. Arpin, S. Brown, S. Ališauskas, G. Andriukaitis, T. Balčiunas, O. D. Mücke, A. Pugzlys, A. Baltuška, B. Shim, S. E. Schrauth, A. Gaeta, C. Hernández-García, L. Plaja, A. Becker,
A. Jaron-Becker, M. M. Murnane, and H. C. Kapteyn, Science 336, 1287 (2012).

[21] A. Čerkić and D. B. Milošević, Phys. Rev. A 75, 013412 (2007).

[22] B. Schütte, P. Ye, S. Patchkovskii, D. R. Austin, C. Brahms, C. Strüber, T. Witting, M. Yu. Ivanov, J. W. G. Tisch, and J. P. Marangos, arXiv:1603.5493.

[23] M. Faubel, S. Schlemmer, and J. P. Toennies, Z. Phys. D 10, 269 (1988).

[24] N. Kurahashi, S. Karashima, Y. Tang, T. Horio, B. Abulimiti, Y.-I. Suzuki, Y. Ogi, M. Oura, and T. Suzuki, J. Chem. Phys. 140, 174506 (2014).

[25] A. Kothe, J. Metje, M. Wilke, A. Moguilevski, N. Engel, R. Al-Obaidi, C. Richter, R. Golnak, I. Y. Kiyan, and E. F. Aziz, Rev. Sci. Instrum. 84, 023106 (2013).

[26] A. Čerkić and D. B. Milošević, Phys. Rev. A 70, 053402 (2004).

[27] R. Kopold, W. Becker, M. Kleber, and G. G. Paulus, J. Phys. B 35, 217 (2002). 